\title{
Influence of inhibitory synapses on the criticality of ex- citable neuronal networks
}

\author{
F S Borges 1,*, P R Protachevicz ${ }^{2,3}$, V Santos $^{2}$, M S Santos ${ }^{3}$, E C Gabrick ${ }^{2}$, K C

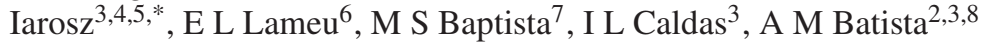 \\ ${ }^{1}$ Center for Mathematics, Computation, and Cognition, Federal University of ABC, 09606-045, São Bernardo \\ do Campo, SP, Brazil. ${ }^{2}$ Graduate Program in Science - Physics, State University of Ponta Grossa, 84030-900, \\ Ponta Grossa, PR, Brazil. ${ }^{3}$ Institute of Physics, University of São Paulo, 05508-900, São Paulo, SP, Brazil. \\ ${ }^{4}$ Faculdade de Telêmaco Borba, 84266-010, Telêmaco Borba, PR, Brazil. ${ }^{5}$ Graduate Program in Chemical \\ Engineering, Federal University of Technology Paraná, 84016-210, Ponta Grossa, PR, Brazil. ${ }^{6}$ Cell Biol- \\ ogy and Anatomy Department, University of Calgary, AB T2N 4N1, Calgary, AB, Canada. ${ }^{7}$ Institute for \\ Complex Systems and Mathematical Biology, University of Aberdeen, AB24 3UE, Aberdeen, Scotland, UK. \\ ${ }^{8}$ Department of Mathematics and Statistics, State University of Ponta Grossa, 84030-900, Ponta Grossa, PR, \\ Brazil. \\ Corresponding author: fernandodasilvaborges@gmail.com, kiarosz@gmail.com.
}

\begin{abstract}
In this work, we study the dynamic range of a neuronal network of excitable neurons with excitatory and inhibitory synapses. We obtain an analytical expression for the critical point as a function of the excitatory and inhibitory synaptic intensities. We also determine an analytical expression that gives the critical point value in which the maximal dynamic range occurs. Depending on the mean connection degree and coupling weights, the critical points can exhibit ceasing or ceaseless dynamics. However, the dynamic range is equal in both cases. We observe that the external stimulus mask some effects of self-sustained activity (ceaseless dynamic) in the region where the dynamic range is calculated. In these regions, the firing rate is the same for ceaseless dynamics and ceasing activity. Furthermore, we verify that excitatory and inhibitory inputs are approximately equal for a network with a large number of connections, showing excitatory-inhibitory balance as reported experimentally.
\end{abstract}

Keywords: inhibitory synapses, dynamic range, self-sustaining dynamics

\section{Introduction}

The relation between stimuli and sensation is one of the main research topics in Psychophysics [1]. Stimulus of different sources and intensities can cause different responses in the sensory system [2]. In the early 19th century, Weber and Fechner proposed that stimuli-response relation correspond to a logarithmic function [3, 4]. In the 1950s, Stevens proposed that stimuli-response relation is given by a power law [5]. Due to physiological and anatomical limitation, the relation between stimuli and response have upper and lower limits. The stimuli difference, between the smaller and bigger sensation, define the dynamic range (DR) associated with its sense [6]. In the context of biological systems, e.g. neuronal networks, the DR corresponds to the ability to differentiate the intensity of external stimulus [7].

The DR is proportional to the logarithm of the ratio between the largest value of the external applied stimulus in which the response is close to saturation of the firing rate and the smallest value of the external applied stimulus in which it is weak to modify 
the firing rate. The human sense of sight can perceive changes in about ten decades of luminosity and the hearing covers twelve decades in a range of intensities of sound pressures [5, 8]. The DR of the human vision plays an important role in the design of display devices [9], where as the hearing case it is relevant to cochlear implants [10].

The DR of a neuronal network increases with the network size until it reaches a saturation value [11]. The increase of the DR value is also associated with the increase of the number of excitatory chemical synapses [12, 13]. Borges et al. [14] reported the complementary effect of chemical and electrical synapses on the enhance of the DR. Protachevicz et al. shown that chemical synapses can enhance DR of the neural network submitted to external stimuli [15].

The mammalian brain is composed of excitatory and inhibitory neurons [16]. The balance between excitation and inhibition plays a crucial role in the transmission of information, signal propagation, and regular firing of neurons in many brain areas [17, [18]. Neuronal networks with excitatory and inhibitory neurons have been considered to describe the dynamics of primary visual cortex [16, 19], cortical firing patterns [20, [21, 22, 23], and synaptic plasticity mechanisms [24, 25, 26, 27].

Kinouchi and Copelli [3] proposed a model of an excitable network based on ErdösRényi (ER) random graphs [28]. They demonstrated that the DR is maximised at the critical point of a non equilibrium phase transition. A theoretical approach to study the effects of network topology on the DR was presented by Larremore et al. [29, 30], in which was considered only excitatory nodes. Pei et al. [31] investigated the collective dynamics of excitatory-inhibitory excitable networks in response to external stimuli. They found that the DR is maximised at the critical point of phase transition which depends only on the excitatory connections.

The spiking dynamics of a network of excitable excitatory nodes resulting from an initial stimulus ceases after a typically short time at a critical point [3]. However, when inhibitory nodes are considered the collective dynamic can become self-sustaining as shown by Larremore et al. [32]. They showed this behaviour considering an additive probabilistic model, where excitatory nodes increase the probability of activation of their neighbours, and inhibitory nodes decrease the probability. In addition, at critical point the collective dynamics can become self-sustainable (ceaseless dynamic) if a fraction of inhibitory nodes is greater than a threshold. However, in their model they did not consider a refractory period and, for this reason, the neuronal firing rate obtained is higher than the experimentally observed. When refractoriness is included in the model, it is possible to obtain the critical point leading to realistic firing patterns [33, 34].

In this work, we investigate the criticality and dynamic range of a cellular automaton modelling a neuronal network in which the neurons are connected by means of excitatory and inhibitory chemical synapses [12, 14]. In order to understand the relationship between maximisation of the DR and the critical self-sustainable activity, we consider a refractory period in the model like the one proposed by Larremore et al. [32]. With the refractory period, the model exhibits more realistic firing rates and critical self-sustained activity. In our simulations, we observe a transition from ceaseless dynamics to ceasing activity when the mean connection degree of the network is increased. We observe that the external stimulus mask effects of self-sustained activity in the region where the DR is calculated, and the firing rate is the same for the ceaseless dynamics and ceasing activity. Furthermore, we obtain an analytical expression 
for the DR as a function of the mean excitatory and inhibitory synaptic intensities. In a network with a large number of connections, we show that the maximal DR value occurs in the critical points where excitatory and inhibitory inputs are approximately equal. In this situation, the neuronal network is in a balanced state. Shew et al. [35] showed experimentally that the DR is maximised when the excitatory and inhibitory synaptic input are balanced. Our work thus provides theoretical explanations for this experimental result.

The paper is organised as follows. In Section 2, we introduce the model. Section 3 presents our analytical results about the dynamic range. In the last Section, we draw our conclusions.

\section{Model}

We consider a $n$ states cellular automaton model composed of $N$ excitable elements. The state of each neuron $i$ is described by the variable $s_{i}(i=1, \ldots, \ldots n)$. In this representation, each neuronal state is associated with the neuronal activity [3, 36]. The resting state is given by $s_{i}=0$, the excited state by $s_{i}=1$, and $s_{i}=2, \ldots, n-1$ are the refractory states. The elements can not be excited during the refractory states. In the model, we consider excitatory and inhibitory neurons [32]. Inhibitory and excitatory inputs are related to the excitatory and inhibitory neurons, respectively. To model the interaction of the synaptic inputs, we considere a probability function. The activation probability of a node in the resting state is given by function $G\left(x_{i}\right)$ [32]

$$
G\left(x_{i}\right)=G\left(\sum_{j=1}^{N} A_{i j} \delta\left(s_{j}(t), 1\right)\right)
$$

where $G\left(x_{i}\right)=0$ for $x_{i} \leq 0, G\left(x_{i}\right)=x_{i}$ for $0<x_{i}<1$, and $G\left(x_{i}\right)=1$ for $x_{i} \geq 1 . G\left(x_{i}\right)$ is a piecewise linear function, known as transfer function, with three pieces. The weighted matrix $\mathbf{A}$ has elements $A_{i j}>0$ for excitatory connections and $A_{i j}<0$ for inhibitory connections. The Kronecker delta $\delta(a, b)$ is equal to 1 when $a=b$ and zero otherwise. The dynamics of both excited and the refractory states are deterministic. If $s_{i}=1$, in the next time steps the state is updated to $s_{i}=2$, and so forth, until $s_{i}=n-1$, returning to the resting state $s_{i}=0$ in the next time step. The fractions of excitatory and inhibitory nodes correspond to $f_{\text {ex }}$ and $f_{\text {in }}$, respectively, and the condition $f_{\text {ex }}+f_{\text {in }}=1$ is always satisfied. In order to simplify the analysis, we arrange the $i$ indexes as $1 \leq i \leq f_{\mathrm{ex}} N$ for excitatory nodes, whereas $f_{\mathrm{ex}} N+1 \leq i \leq N$ for inhibitory ones. Fig. 1 displays an schematic illustration of (a) the neuronal dynamics for $n=3$ states, (b) a neuron receiving chemical synaptic inputs and (c) the function $G\left(x_{i}\right)$ as a function of the sum of all synaptic inputs.

The neuronal response at a given time $t$ can be quantified using the density of spiking neurons

$$
p(t)=\frac{1}{N} \sum_{i=1}^{N} \delta\left(s_{i}(t), 1\right),
$$

which is interpreted as the probability for a random neuron to be in the excited state at 
(a)

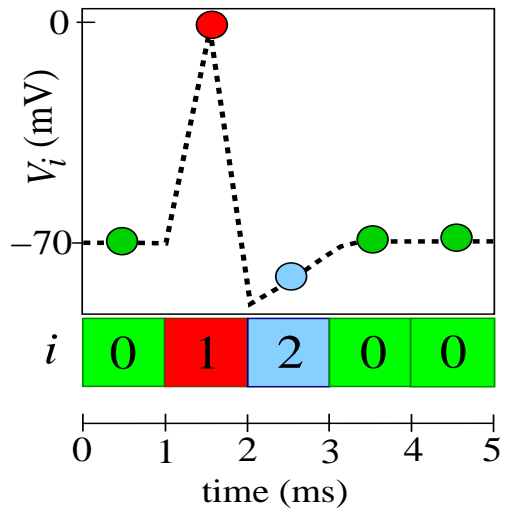

(b)

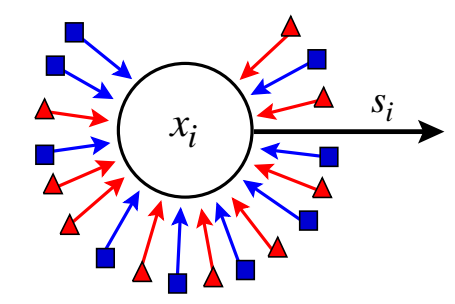

(c)

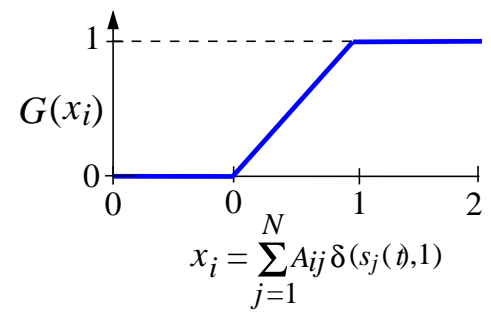

Figure 1: Representation of the neuronal activity by a cellular automaton with $n=3$ states. (a) Illustrative membrane potential for each neuron $i$, where $s_{i}$ represent the rest $\left(s_{i}=0\right)$, the active $\left(s_{i}=1\right)$, and refractory $\left(s_{i}=2\right)$ states. (b) Chemical synaptic inputs arriving in the neuron $i$. Red triangles and blue squares represent the excitatory and inhibitory inputs, respectively. (c) The neuronal activation probability $\left(G\left(x_{i}\right)\right)$ is given by a function of all chemical inputs arriving in the neuron $i$ at time $t$. 
time $t$. With the time series of $p(t)$, we calculate the average firing rate

$$
F=\overline{p(t)}=\frac{1}{T} \sum_{t=1}^{T} p(t)
$$

where $T$ is the time window chosen to calculate the average.

In this work, we consider random networks and for this case the update equations are the same for both excitatory and inhibitory nodes [31]. Our networks are built according to the Erdös-Rényi random graphs with probability equal to $K /(N-1)$, where $K$ is the average degree of connections of the network. Assuming that the events of the neighbours of an excited node are statistically independent for large $t$, we obtain the following mean field map for the density of spiking neurons

$$
p(t+1)=[1-(n-1) p(t)](\eta+G(x)-\eta G(x)),
$$

where the external stimulus $\eta=1-\exp (-r \Delta t)$ is a Poisson process with mean perturbation rate $r$ in the time interval $\Delta t$ [3]. In our simulations, we use $\Delta t=1$.

Setting the weights $A_{i j}=S_{\mathrm{ex}}$ for the excitatory connections and $A_{i j}=-S_{\text {in }}$ for the inhibitory ones, when the network reaches a stationary state, the mean value of $x_{i}$ is given by

$$
\langle x\rangle=f_{\mathrm{ex}} K S_{\mathrm{ex}} p(t)-f_{\text {in }} K S_{\text {in }} p(t) .
$$

Defining $\sigma_{\mathrm{ex}}=K S_{\mathrm{ex}}$ and $\sigma_{\mathrm{in}}=K S_{\mathrm{in}}$, we obtain

$$
\langle x\rangle=\left(f_{\mathrm{ex}} \sigma_{\mathrm{ex}}-f_{\text {in }} \sigma_{\text {in }}\right) p(t) .
$$

In the stationary state we have $p(t+1)=p(t)=p^{*}$ and $F \approx p^{*}$. Substituting in Eq. (4), and considering the case of no external perturbation $(\eta=0)$, we get

$$
F_{0}=\left(1-(n-1) F_{0}\right) G(x) \text {. }
$$

In the regime $0<\left(f_{\text {ex }} \sigma_{\text {ex }}-f_{\text {in }} \sigma_{\text {in }}\right) F<1$, the model implies $G(x)=x$, and therefore

$$
F_{0}=\left(1-(n-1) F_{0}\right)\left(f_{\mathrm{ex}} \sigma_{\mathrm{ex}}-f_{\text {in }} \sigma_{\mathrm{in}}\right) F_{0} .
$$

Solving for $F_{0}$ we get

$$
F_{0}=\frac{1-\left(f_{\mathrm{ex}} \sigma_{\mathrm{ex}}-f_{\mathrm{in}} \sigma_{\mathrm{in}}\right)^{-1}}{n-1}
$$

There is a phase transition from ceasing activity $\left(F_{0}=0\right)$ to ceaseless activity $\left(F_{0}>0\right)$. In the critical point of this phase transition $\left(F_{0} \rightarrow 0\right)$, we obtain

$$
\sigma_{\text {in }}=\frac{f_{\mathrm{ex}} \sigma_{\mathrm{ex}}-1}{f_{\text {in }}} .
$$

This relation shows that the critical point in the model is given by $f_{\mathrm{ex}} \sigma_{\mathrm{ex}} \geq 1$, implying the necessity of a minimum fraction of excitatory neurons. In addition, we observe that $f_{\mathrm{ex}} \sigma_{\mathrm{ex}} \approx f_{\text {in }} \sigma_{\text {in }}$ for $\sigma_{\mathrm{ex}} \gg 1$. Then, for a highly connected network $(\sigma \propto K)$, we obtain approximately the same amount of excitatory and inhibitory mean inputs from 

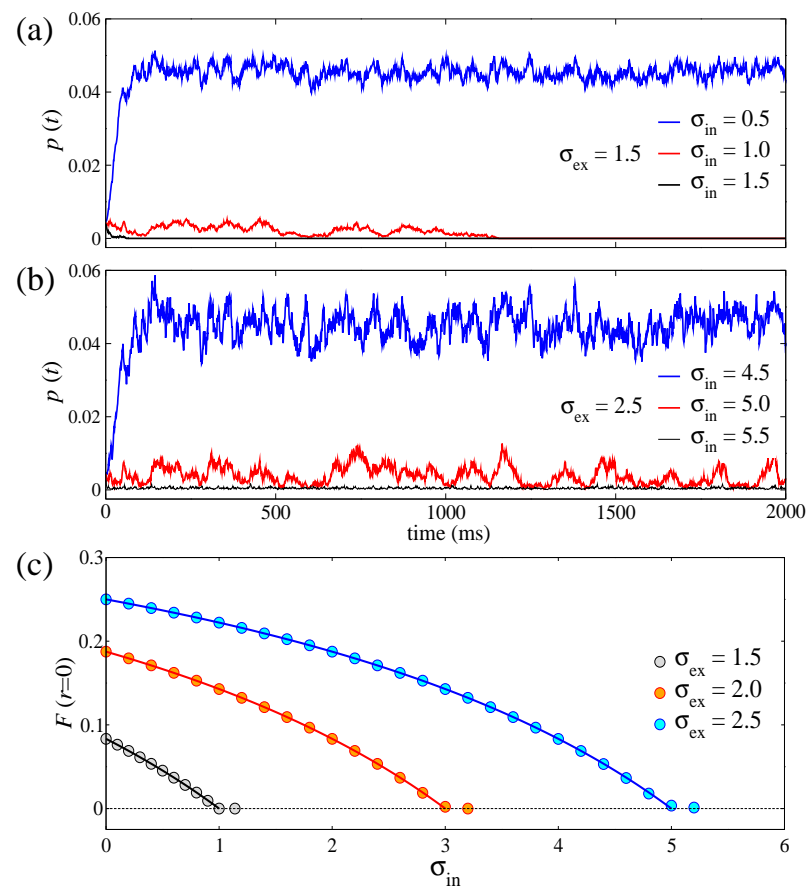

Figure 2: Time series of the density of spiking neurons for subcritical (black line), critical (red line), and supercritical (blue line) values of $\sigma_{\text {in }}$ for (a) $\sigma_{\text {ex }}=1.5$ and (b) $\sigma_{\mathrm{ex}}=2.5$. In (c), we plot the average firing rate as a function of $\sigma_{\mathrm{in}}$ for $\sigma_{\mathrm{ex}}=1.5$ (black circles), $\sigma_{\mathrm{ex}}=2.0$ (red circles) and $\sigma_{\mathrm{ex}}=2.5$ (blue circles). The points are obtained from numerical simulations while the curves are given by Eq. 9 The parameters are $N=10^{5}, K=10^{4}, r=0, n=3$, and $f_{\text {ex }}=0.8$. 
probabilistic synapses. In this situation, our model exhibits a state which is critical and balanced.

In this work, we split in three theoretical firing regimes that depende of Eq. 9 and of the parameters $f_{\mathrm{ex}}, \sigma_{\mathrm{ex}}, f_{\mathrm{in}}$, and $\sigma_{\mathrm{in}}$. (i) if $F_{0}<0$ we have a subcritical regime; (ii) if $F_{0}=0$ we have a critical regime; and (iii) if $F_{0}>0$ we have a supercritical regime. In Figs. 2a) and 2(b), we show the density of spiking neurons without external perturbation as a function of the time for values of $\sigma_{\text {in }}$ in the subcritical, critical, and supercritical regime for different values of $\sigma_{\mathrm{ex}}$. We choose randomly $0.4 \%$ of neurons to be active $\left(s_{i}=1\right)$ at $t=0$. In Fig. 2(c), we show the relation between $F_{0}$ and $\sigma_{\text {in }}$ for some values of $\sigma_{\mathrm{ex}}$. We verify that the theoretical results given by Eq. 9 are in agreement with our numerical simulations.

When a great number of neurons presents $x_{i}<0$, even at the critical point, in the numerical simulations $F$ can be positive for a large time span. In Fig. 2, we see that the spiking activity ceases rapidly when $\sigma_{\mathrm{ex}}=1.5$, whereas it is persistent at the critical point when $\sigma_{\mathrm{ex}}=2.5$. We verify that the activity is not persistent if we increase the average degree of connections. Fig. 3(a) exhibits the density of spiking neurons considering $K=2 \times 10^{4}$, for subcritical, critical (three different initial conditions) and supercritical values of $\sigma_{\text {in }}$. In Fig. 3 b), we plot the distribution of $x_{i}$ for 1000 time steps, $N=10^{5}, K=10^{4}$ (blue), and $K=2 \times 10^{4}$ (red). In both cases, we find $\langle x\rangle$ and $F \approx 0.0031$. In the first case, approximately $2.18 \%$ of $x_{i}$ present negative values. In the second case, about $5.00 \%$ of $x_{i}$ are less than zero. We observe that greater values of $S_{\mathrm{ex}}=\sigma_{\mathrm{ex}} / K$ and $S_{\text {in }}=\sigma_{\text {in }} / K$ contribute for the persistent activity at the critical point.
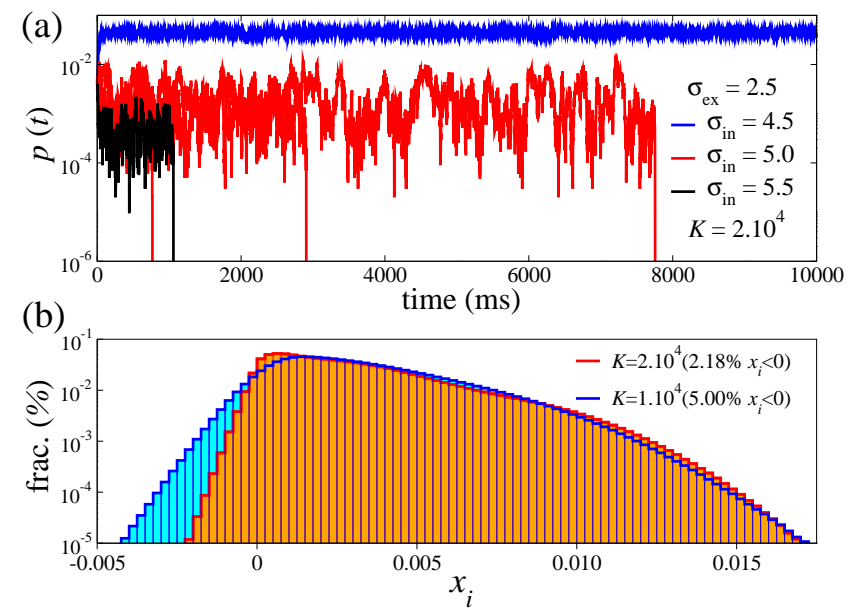

Figure 3: (a) Time series of the density of spiking neurons for the subcritical (black line), critical (red line), and supercritical (blue line) values of $\sigma_{\text {in }}$ with $\sigma_{\mathrm{ex}}=2.5$. (b) Distribution of $x_{i}$ values for the average degree of connections $K=2 \times 10^{4}$ (red) and $K=1 \times 10^{4}$ (blue). Parameters are $N=10^{5}, f_{\mathrm{ex}}=0.8, \sigma_{\mathrm{ex}}=1.5$, and $\sigma_{\mathrm{ex}}=2.5$. 


\section{Dynamic Range (DR)}

The behaviour of the average firing rate $(F)$ as a function of the external stimulus $(r)$ shows a minimum and a maximum saturation ( $F_{0}$ and $F_{\max }$, respectively) for a range of $r$ values, as shown in Fig. 4. The DR is defined as

$$
\Delta=10 \log _{10} \frac{r_{\text {high }}}{r_{\text {low }}},
$$

where $\Delta$ is the stimulus interval (measured in $\mathrm{dB}$ ) in which changes in $r$ can be perceived as changes in $F$, and it is between the disregarding stimuli that cause a response small to be distinguished from $F_{0}$ and the saturation $F_{\max }[3]$. The interval $\left[r_{\text {low }}, r_{\text {high }}\right]$ is found from its correspondent in $F$, $\left[F_{\text {low }}, F_{\text {high }}\right]$, where $F_{\text {high }}=F_{0}+0.95\left(F_{\max }-F_{0}\right)$ and $F_{\text {low }}=F_{0}+0.05\left(F_{\max }-F_{0}\right)$.

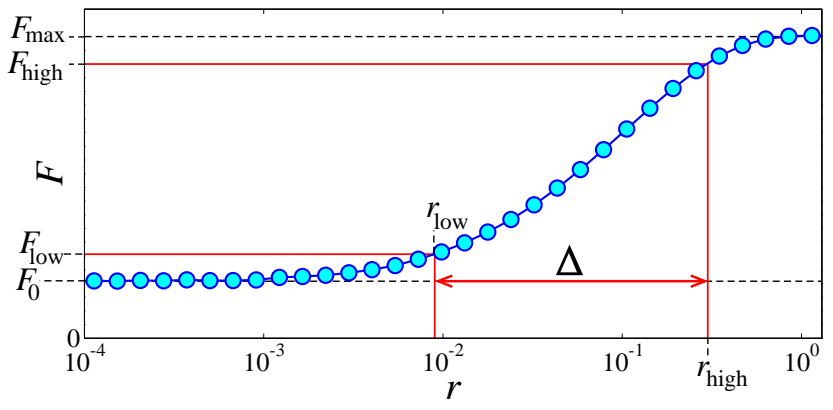

Figure 4: Mean firing rate as a function of intensity stimuli.

For $0<\left(f_{\mathrm{ex}} \sigma_{\mathrm{ex}}-f_{\text {in }} \sigma_{\text {in }}\right) F<1$ (in the stationary state) we approximate Eq. 4 as

$$
\begin{aligned}
F & =[1-(n-1) F]\left[\eta+\left(f_{\mathrm{ex}} \sigma_{\mathrm{ex}}-f_{\min } \sigma_{\mathrm{in}}\right) F\right. \\
& \left.-\left(f_{\mathrm{ex}} \sigma_{\mathrm{ex}}-f_{\mathrm{in}} \sigma_{\mathrm{in}}\right) \eta F\right] .
\end{aligned}
$$

Rearranging the terms, we obtain

$$
\begin{aligned}
& {\left[(n-1)\left(f_{\mathrm{ex}} \sigma_{\mathrm{ex}}-f_{\mathrm{min}} \sigma_{\mathrm{in}}\right)(1-\eta)\right] F^{2} } \\
+\quad & {\left[1+(n-1) \eta-\left(f_{\mathrm{ex}} \sigma_{\mathrm{ex}}-f_{\min } \sigma_{\mathrm{in}}\right)(1-\eta)\right] F } \\
-\quad & \eta=0 .
\end{aligned}
$$

As $\eta$ depends on $r$, by solving Eq. 13, we are able to determine the dependence of the average firing rate on the mean perturbation rate $r$, as well as its dependence on all the parameters of the network.

In Fig. 5a), we plot $F$ as a function of $r$ for subcritical, critical and supercritical values of $\sigma_{\text {in }}$. The lines represent the theoretical values from the solution of expression 13 and the symbols are obtained through numerical simulations. In the inset of Fig. 5 (a), we show a magnification to demonstrate that there are diferences between the theoretical and the numerical values of $F$ for $r$ values out of the region where DR is calculated (green). 
For a cellular automaton with $n$ states, the maximum average firing rate is given by $F_{\max }=1 / n$. Deriving $F_{0}$ in Eq. 9, $F_{\text {low }}$ and $F_{\text {high }}$ can be obtained. Then, $\eta_{\text {low }}$ and $\eta_{\text {high }}$ can be calculated directly by

$$
\eta_{\text {low,high }}=\frac{\lambda F_{\text {low,high }}}{1-\lambda F_{\text {low,high }}}\left[\frac{1}{\lambda-(n-1) \lambda F_{\text {low,high }}}-1\right],
$$

where we substitute $\lambda=f_{\mathrm{ex}} \sigma_{\mathrm{ex}}-f_{\text {in }} \sigma_{\text {in }}$ for convenience. Now we calculate $r_{\text {low }}$ and $r_{\text {high }}$ according to

$$
r_{\text {low,high }}=-\ln \left|1-\eta_{\text {low,high }}\right| .
$$

Using the equations 9, 14, 15, and the expressions for $F_{\max }, F_{\text {low }}$, and $F_{\text {high }}$, we calculate the dynamic range.
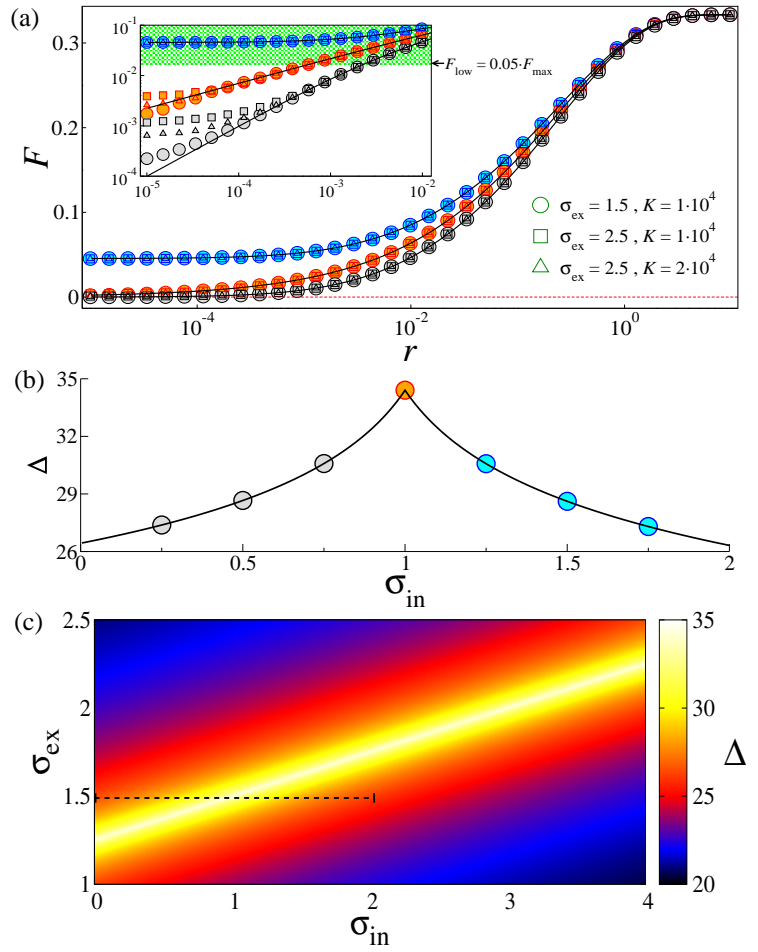

Figure 5: (a) Average firing rate $(F)$ as a function of the mean perturbation rate $(r)$. The black, red, and blue symbols correspond to subcritical, critical and supercritical values of $\sigma_{\text {in }}$, respectively. (b) Dynamic range as a function of $\sigma_{\text {in }}$ for $\sigma_{\text {ex }}=1.5$. The coloured circles are obtained by means of simulations and the black lines represent the theoretical results from the analytical expression. Dynamic range of Fig. (b) is indicated on the $\sigma_{\mathrm{ex}} \times \sigma_{\text {in }}$ parameter space by a dash line in Fig. (c). We consider $N=10^{5}, K=10^{4}$, and $f_{\text {ex }}=0.8$.

In Fig. 5(b), we compare our numerical and the theoretical results. We verify that the maximum DR occurs for $\sigma_{\text {in }}=1$, which is the critical point for the considered 
parameters $\left(\sigma_{\mathrm{ex}}=1.5\right)$. In Fig. $\left.5 \mathrm{c}\right)$, the color scale represents the value of DR for each pair $\left(\sigma_{\mathrm{ex}}, \sigma_{\mathrm{in}}\right)$. The dashed line indicates the range taken in (b). From the figure, we see that the maximum value of DR follows the line given by the critical point expression $\sigma_{\mathrm{in}}=4 \sigma_{\mathrm{ex}}-5$ (Eq. 10). Since the excitatory-inhibitory ratio is 4 , the mean input approaches zero as the $\sigma_{\mathrm{ex}}$ value increases. Therefore, the model shows both the critical and balanced state in a network with a great number of connections, where the weights are not small. For instance, for $K=2 \times 10^{4}$ and $S_{\text {ex }}=0.5$, we obtain $\sigma_{\mathrm{ex}}=K S_{\mathrm{ex}}=10^{4}$ and the critical $\sigma_{\text {in }}=4 \times 10^{4}-5$. The ration between excitatory and inhibitory input is $\frac{4 \times \sigma_{\mathrm{ex}}}{\sigma_{\mathrm{in}}} \approx 1.0013$. In this situation, the DR is maximum and closes to a balanced state.

\section{Conclusions}

The firing dynamics of a network of excitable excitatory nodes resulting from an initial stimulus ceases after a typically short time at a critical point. However, when inhibitory nodes are considered the collective dynamic can become self-sustained. In this work, we build a cellular automaton model with excitatory and inhibitory connections. In our network, we consider that the connections have different weights. We find an expression that relates the mean of excitatory and inhibitory weights at the critical point. We also calculate an expression for the dynamic range and show that at the critical point it reaches its maximal value.

Depending of mean connection degree and coupling weights, the critical points can exhibit ceasing or ceaseless dynamics (self-sustained activity). However, the dynamic range is equal in both cases. We observe that the external stimulus mask some effects of self-sustained activity in the region where the DR is calculated. In these regions, the firing rate is the same for ceaseless dynamics and ceasing activity. Furthermore, we show that at the critical point the amount of excitatory and inhibitory inputs can be approximately equal in a densely connected network. This result showing excitatoryinhibitory balanced was experimentally reported by Shew et al. [35].

In future works, we plan to consider other network topologies, such as small-world and scale-free, to study the influence of inhibitory synapses on the criticality of excitable neuronal networks.

\section{Acknowledgment}

This study was possible by partial financial support from the following Brazilian government agencies: Fundação Araucária, National Council for Scientific and Technological Development, Coordination for the Improvement of Higher Education Personnel, and São Paulo Research Foundation (2015/07311-7, 2017/18977-1, 2018/03211-6, 2020/04624-2). 


\section{References}

[1] G A Chescheider. Psychophysics: The Fundamentals 3th ed., Psychology Press (2013)

[2] S Stevens. Psychophysics. Transaction Publisher (1975)

[3] O Kinouchi, M Copelli. Nature Physics 2 348-351 (2006)

[4] A Levina, J M Herrmann, T Geisel. Nature Physics, Nature Publishing Group 3 857-869 (2007)

[5] S S Stevens, Psychophysics: Introduction to its perceptual neural and social prospects, Transaction Publisher, New Jersey (2008)

[6] D J Murray, Behav. Brain Sci. 16 115-186 (1993)

[7] L L Gollo, O Kinouchi, M Copelli. PLoS Comput. Biol. 5 e1000402 (2009)

[8] D R Chialvo. Nat. Phys. 2 301-302 (2006)

[9] E Reinhard, G Ward, S Pattanaik, P Debevec, W Heidrich, K Myszkowski. High Dynamic Range Imaging: Acquisition, Display and Image-Based Lighting 2nd ed., Morgan Kaufmann Publishers, Burlington, USA (2010)

[10] A J Spahr, M F Dorman, L H Loiselle. Ear Hear. 28 260-275 (2007)

[11] C A S Batista, R L Viana, S R Lopes, A M Batista. Phys. A 10 628-640 (2014)

[12] R L Viana, F S Borges, K C Iarosz, A M Batista, S R Lopes, I L Caldas. Commun. Nonlinear Sci. Numer. Simul. 19 164-172 (2014)

[13] K C Iarosz, A M Batista, R L Viana, S R Lopes, I L Caldas, T J P Penna. Physica A 391, 819-827 (2012)

[14] F S Borges, E L Lameu, A M Batista, K C Iarosz, M S Baptista, R L Viana. Physica A 430 236-241 (2015)

[15] P R Protachevicz,F S Borges, K C Iarosz, I L Caldas, M S Baptista, R L Viana, E L Lameu, E E N Macau, A M Batista. Physica A 492 1045-1052 (2018)

[16] Y Adini, D Sagi, M Tsodyk. Proc. Natl. Acad. Sci. USA 9410426 (1997)

[17] E R Kandel, J H Schwartz, T M Jessell. Principles of Neural Science 4th ed., McGraw-Hill Companies, USA (2000)

[18] G Buzsaki, Rhythms of the Brain, Oxford University Press, Oxford (2006)

[19] M Kurant, P Thiran. Phys. Rev. Lett. 96138701 (2006)

[20] F S Borges, P R Protachevicz, E L Lameu, R C Bonetti, K C Iarosz, I L Caldas, M S Baptista, A M Batista. Neural Netw. 901 (2017) 
[21] P R Protachevicz, R R Borges, A S Reis, F S Borges, K C Iarosz, E L Lameu, E E N Macau, R L Viana, I M Sokolov, F A S Ferrari, J Kurths, A M Batista, C-Y Lo, Y He, C-P Lin. Physiol. Meas. 39074006 (2018)

[22] F S Borges, P R Protachevicz, R F O Pena, E L Lameu, G S V Higa, A H Kihara, F S Matias, C G Antonopoulos, R de Pasquale, A C Roque, K C Iarosz, P Ji, A M Batista. Physica A 537122671 (2020)

[23] P R Protachevicz, F S Borges, E L Lameu, P Ji, K C Iarosz, A H Kihara, I L Caldas, J D Szezech Jr, M S Baptista, E E N Macau, C Antonopoulos, A M Batista, J Kurths. Front. Comput. Neurosc. 131 (2019)

[24] R R Borges, F S Borges, E L Lameu, A M Batista, K C Iarosz, I L Caldas, C G Antonopoulos, M S Baptista, Neural Netw. 88, 58-64 (2017)

[25] R R Borges, F S Borges, E L Lameu, P R Protachevicz, K C Iarosz, I L Caldas, R L Viana, E E N Macau, M S Baptista, C Grebogi, A. M. Batista. Braz. J. Phys. 47, 678-688 (2017)

[26] E L Lameu, S Yanchuk, E E N Macau, F S Borges, K C Iarosz, I L Caldas, P R Protachevicz, R R Borges, R L Viana, J D Szezech Jr, A M Batista, J Kurths. Chaos 28, 085701 (2018)

[27] E L Lameu, E E N Macau, F S Borges, K C Iarosz, I L Caldas, R R Borges, P R Protachevicz, R L Viana, A M Batista. Eur. Phys. J. Spec. Top. 227, 673-682 (2018)

[28] P Erdös; A Rényi. On Random Graphs. Publicationes Mathematicae 6 290-297 (1959)

[29] D B Larremore, W L Shew, J G Restrepo. Phys. Rev. Lett. 106058101 (2011)

[30] D B Larremore, W L Shew, E Ott, J G Restrepo. Chaos 21025117 (2011)

[31] S Pei, S Tang, S Yan, S Jiang X Zhang, Z Zheng. Physical Review E 86021909 (2012)

[32] D B Larremore, W L Shew, E Ott, F Sorrentino, J G Restrepo. Phys. Rev. Lett. 112138103 (2014)

[33] I L D Pinto, M Copelli. Phys. Rev. E 100062416 (2019)

[34] M Girardi-Schappo, L Brochini, A A Costa, T T A Carvalho, O Kinouchi. Phys. Rev. Res. 2012042 (2020)

[35] W L Shew, H Yang, T Petermann, R Roy, D Plenz. J. Neurosci. 291559515600 (2009)

[36] M Copelli, A C Roque, R F Oliveira, O Kinouchi. Phys. Rev. E 65060901 (2002) 